\title{
Dynamics of Electron Wave Propagation in Photoionization Microscopy: I. Semiclassical Open-Orbit Theory
}

\author{
L. B. Zhao \\ William \& Mary \\ John B. Delos \\ William \& Mary, jbdelos@wm.edu
}

Follow this and additional works at: https://scholarworks.wm.edu/aspubs

Part of the Physics Commons

\section{Recommended Citation}

Zhao, L. B. and Delos, John B., Dynamics of Electron Wave Propagation in Photoionization Microscopy: I. Semiclassical Open-Orbit Theory (2010). Physical Review A, 81(5).

https://doi.org/10.1103/PhysRevA.81.053417

This Article is brought to you for free and open access by the Arts and Sciences at W\&M ScholarWorks. It has been accepted for inclusion in Arts \& Sciences Articles by an authorized administrator of W\&M ScholarWorks. For more information, please contact scholarworks@wm.edu. 


\title{
Dynamics of electron wave propagation in photoionization microscopy. I. Semiclassical open-orbit theory
}

\author{
L. B. Zhao and J. B. Delos \\ Department of Physics, College of William \& Mary, Williamsburg, Virginia 23187, USA
}

(Received 8 April 2010; published 20 May 2010)

\begin{abstract}
This is the first of two papers that develop theories and numerical methods for photoionization microscopy of hydrogen atoms in strong electric fields, in semiclassical and quantum-mechanical frameworks, respectively. In this paper, semiclassical open-orbit theory is presented to describe the propagation of outgoing electron waves to macroscopic distances. Spatial distributions of electron probability densities and current densities are predicted. The open-orbit theory, based on an assumption that electron waves propagate along classical paths from a pointlike source to a detector, provides a clear and intuitive physical picture to interpret structures of observed geometrical interference patterns in photoionization microscopy. We calculate photoelectron ejection of hydrogen atoms in electric fields, and comparison is made with quantum-mechanical results, which will be detailed in the second paper [Zhao and Delos, Phys. Rev. A 81, 053418 (2010)]. A strong quantum tunneling effect has been found. Such a tunneling effect should be visible in the experiment.
\end{abstract}

DOI: 10.1103/PhysRevA.81.053417

PACS number(s): $32.80 . \mathrm{Fb}, 03.65 . \mathrm{Sq}, 07.81 .+\mathrm{a}$

\section{INTRODUCTION}

Photodetachment and photoionization microscopy were first proposed in the 1980s by Demkov et al. [1] and Kondratovich and Ostrovsky [2]. In these proposed experiments, an electron of a fixed energy is detached from a negative ion or is ionized from an atom in a static applied field. The electron is accelerated by the field to a position-sensitive detector. The electron waves traveling along multiple paths from the negative ion or the atom to a point on the detector interfere to produce oscillatory patterns.

The first experimental implementation of photodetachment microscopy was made by Blondel et al. [3]. Ejected electrons produced by photodetachment of $\mathrm{Br}^{-}$in electric fields were recorded on a high resolution detector perpendicular to the applied electric field. The recorded pattern displayed concentric interference fringes. A subsequent observation of photodetachment of $\mathrm{O}^{-}$in electric fields was also performed in the same group [4]. The experimental results were supported by the quantum calculation of Kramer et al. [5]. The comparison shows excellent agreement. Photodetachment microscopy opens a new way to measure electron affinities of neutral atoms by means of interference patterns [6-8] with accuracy higher than any current $a b$ initio calculations for multielectron systems [9].

A semiclassical method was developed by $\mathrm{Du}$ [10] to predict outgoing electron current distributions in photodetachment of $\mathrm{H}^{-}$in electric fields prior to the experiment of Blondel et al. [3]. The method of Du may be extended to treat more complicated negative ions. Recently, an investigation of the electron dynamics in parallel electric and magnetic fields has been reported by Bracher and Delos [11]. The semiclassical analysis of photodetachment of $\mathrm{H}^{-}$shows that the simultaneous presence of electric and magnetic forces leads to intricate shapes of refracted electron waves, and also the quantum solution is best understood by being compared with classical electron motion. A separate semiclassical result for $\mathrm{H}^{-}$was also presented by Gao et al. [12]. The effects of bifurcations on the ejected electron current distributions were discussed.
The first experimental implementation of photoionization microscopy was made by Nicole et al. [13]. It was found that the interference patterns evolve smoothly with the excess energy above the saddle point. The observed patterns were partially explained with the semiclassical theory of Kondratovich and Ostrovsky [2]. A more detailed semiclassical analysis was presented by Bordas et al. [14]. In contrast with the case of photodetachment, in which only two trajectories interfere at each given point on a detector, for photoionization, an infinite number of electron trajectories contribute to the observed interference. The simultaneous presence of Coulomb and electric fields makes descriptions of ejected electron motions complicated. The semiclassical theory used by Bordas et al. is incomplete. They did not incorporate Maslov indices, did not treat tunneling into classically forbidden regions, and did not correct singularities that arise in semiclassical approximations. However, after making some adjustments related to uncertainties in the electric-field strengths, they got a decent (but not really a quantitative) comparison between theory and experiment.

Inspired by their work, we present, in this paper, a semiclassical open-orbit theory of photoionization microscopy for hydrogen atoms in constant applied electric fields. We include effects of Maslov indices, which significantly alter the spatial distribution of the current densities, and repair singularities at caustics and cylindrical foci by means of Airyfunction and Bessel-function approximations. Furthermore, we use Bohr-Sommerfeld quantization conditions to predict resonance positions of hydrogen atoms in electric fields.

The paper is organized as follows. In Sec. II, we introduce the semiclassical open-orbit theory, illustrate the physical picture, and outline main theoretical formulas to calculate electron probability densities and current densities. Section III gives the numerical procedures to solve Hamiltonian equations along trajectories in combined Coulomb and electric fields. In Sec. IV, we outline the uniform approximation and the Besselfunction methods, in which singularities of semiclassical wave functions can be fixed. In Sec. V, we present predictions of electron probability densities and electron current probabilities 
at a detector resulting from photoionization of hydrogen atoms in some electric-field strengths from an initial $n=2$ state, and comparison is made with quantum-mechanical calculations. Finally, Sec, VI summarizes the main conclusions.

\section{OPEN-ORBIT THEORY}

\section{A. Physical picture, open orbits, and bound orbits}

In this section, we describe the physical picture of the outward propagation of electron waves generated in nearthreshold photoionization of hydrogen atoms in uniform electric fields, and note both open orbits and bound orbits. When light irradiates hydrogen atoms in an electric field, the electrons of the atoms may absorb a photon and jump to a high-energy state. This is a quantum process. The energy of the outgoing electrons may be sharply defined, but their initial direction of motion is not; the initial angular distribution is given by an angular factor $\mathcal{Y}_{m}(\theta)$ [see Eq. (3)] in the wave function. As the electron moves away from the nucleus, the wave functions can be constructed using the semiclassical approximation, following classical trajectories. The electrons traveling along some of the outgoing trajectories escape from the atoms, and, as a consequence, the waves propagate to a large distance where a position-sensitive detector is placed. We call such trajectories open orbits. Whenever two or more open orbits arrive at a given point on the detector, the corresponding waves interfere constructively or destructively, and an observable interference pattern is produced on the detector. A schematic is shown in Fig. 1. Not all the trajectories reach the detector. The electrons traveling along some of the outgoing classical trajectories never escape from the atoms. There exists a critical angle (Sec. III D) such that the electrons leaving the atoms below (i.e., in a downhill sense from) the critical angle reach the detector, but those going out

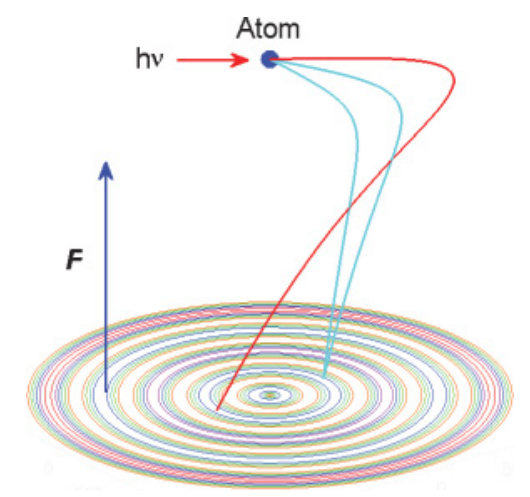

FIG. 1. (Color online) Schematic of propagation of electrons in combined Coulomb and electric fields. The detector is placed under the source of $\mathrm{H}$ atoms. The electric field $\mathscr{F}$ is upward along the $z$ axis, so the field force is downward. $\mathrm{H}$ atoms are irradiated by a laser light beam, and an electron of an atom may absorb a photon to be ionized. The waves of the ionized electrons propagate outward in all directions, following classical trajectories. If two trajectories reach the same point in space, the associated waves interfere constructively or destructively. This interference pattern is recordable on a positive-sensitive detector. Here, two of three trajectories reach the same point on a detector, and a set of concentric interference fringes is schematically plotted. above (i.e., uphill from) the critical angle stay forever in the vicinity of the atoms. In contrast with open orbits, we call the trajectories, which never escape from the atoms, bound orbits. The closed orbits [15], which go out from and then return to the atomic source, are a special set of the bound orbits. The bound orbits satisfying the Bohr-Sommerfeld quantization condition are related to quasibound or resonance quantum states. These resonance states also have a substantial effect on the interference pattern. We will give estimates of their locations by semiclassical formulas, and calculate their effects on interference patterns by a fully quantum-mechanical method described in the accompanying paper.

\section{B. Outgoing waves}

To calculate wave functions of electrons in combined Coulomb and electric fields, we divide the configuration space into inner and outer regions, as is done in closed-orbit theory [15]. In the inner region, within $\sim 10-100 a_{0}$ from the nucleus, the interaction of electrons with the Coulomb field is much stronger than its interaction with the electric field, so the latter can be neglected, and, thus, the wave functions can be evaluated with a field-free quantum-mechanical method. The result is $[15,16]$

$$
\psi_{\text {out }}(\boldsymbol{r})=\mathcal{C} \sum_{m} \frac{\exp (i \sqrt{8 r})}{r^{3 / 4}} \mathcal{Y}_{m}(\theta) e^{i m \varphi},
$$

with

$$
\begin{gathered}
\mathcal{C}=-i \pi^{1 / 2} 2^{3 / 4} e^{-i 3 \pi / 4}, \\
\mathcal{Y}_{m}(\theta)=\sum_{\ell}(-1)^{\ell} Y_{\ell m}(\theta, 0) \\
\times \int Y_{\ell m}^{*}\left(\theta^{\prime}, \varphi^{\prime}\right) \frac{J_{2 \ell+1}\left(\sqrt{8 r^{\prime}}\right)}{\sqrt{r^{\prime}}} D \Psi_{\text {ini }}\left(\boldsymbol{r}^{\prime}\right) d \boldsymbol{r}^{\prime},
\end{gathered}
$$

where $J_{2 \ell+1}\left(\sqrt{8 r^{\prime}}\right)$ is the Bessel function, $D$ is the dipole operator, and $\Psi_{\text {ini }}\left(\boldsymbol{r}^{\prime}\right)$ is the initial state of hydrogen atoms. A complete theoretical derivation of the formula has been made by $\mathrm{Du}$ and Delos [15]. Several examples of the form of $\mathcal{Y}_{m}(\theta)$ to treat hydrogen, pseudohydrogen, and sodium atoms from various initial states have been given in Ref. [16].

In the outer region, where the interaction with the electric field is no longer negligible and the motion of the electrons is affected by both the Coulomb force and the electric-field force, the wave function is calculated using a semiclassical approximation. We propagate the wave function outward beginning from a spherical surface dividing the configuration space into the inner and outer regions. On the spherical surface, the outgoing wave is simply obtained from Eq. (1) by taking the spherical radius $r=r_{0} \sim 10-100 a_{0}$. The final result is independent of the radius of that initial sphere. Let $\boldsymbol{q}$ represent the coordinates of the electron on the detector, and let $\boldsymbol{q}_{0}$ represent its coordinates on the initial surface. The semiclassical wave function is written in the form

$$
\Psi(\boldsymbol{q})=\sum_{j} \psi_{\text {out }}^{j}\left(\boldsymbol{q}_{0}\right) A_{j}(\boldsymbol{q}) e^{i\left[S_{j}(\boldsymbol{q})-\mu_{j} \pi / 2\right]},
$$

where the summation runs over all trajectories that arrive at the point $\boldsymbol{q}$; each trajectory begins at a point $\boldsymbol{q}_{0}$ on the 
initial spherical surface, $S_{j}(\boldsymbol{q})$ is the classical action on the $j$ th trajectory,

$$
S_{j}(\boldsymbol{q})=\int_{\boldsymbol{q}_{0}}^{\boldsymbol{q}} \boldsymbol{p} \cdot d \boldsymbol{q}
$$

$A_{j}(\boldsymbol{q})$ is the classical amplitude,

$$
A_{j}(\boldsymbol{q})=\left[J\left(t_{0}, \boldsymbol{q}_{0}\right) / J(t, \boldsymbol{q})\right]^{1 / 2},
$$

with $J$ being a Jacobian defined in the following, and $\mu_{j}$ being the Maslov index for the $j$ th trajectory.

\section{Probability density and electron current distribution}

A position-sensitive detector, on which the ejected electrons are experimentally recorded, is placed under the atomic source in a uniform electric field (Fig. 1). The probability density or the electron current distribution may be calculated on the detector for comparison with the experiment. If the the detector is perpendicular to the $z$ axis, then in cylindrical coordinates $(\rho, z, \varphi)$, the probability density is

$$
\mathcal{P}\left(\rho, z_{\mathrm{det}}, \varphi\right)=\left|\Psi\left(\rho, z_{\mathrm{det}}, \varphi\right)\right|^{2},
$$

and the probability density integrated over an azimuthal angle $\varphi$ is

$$
\mathcal{P}\left(\rho, z_{\mathrm{det}}\right)=\int_{0}^{2 \pi}\left|\Psi\left(\rho, z_{\mathrm{det}}, \varphi\right)\right|^{2} \rho d \varphi .
$$

The electron current distribution, or the differential cross section, may be determined by [10]

$$
\frac{d \sigma(\boldsymbol{r})}{d s}=\frac{2 \pi \omega}{c} \boldsymbol{j} \cdot \boldsymbol{n},
$$

where $\omega$ is the photon frequency, $c$ is the speed of light, $\boldsymbol{n}$ is a unit vector normal to the screen at $\boldsymbol{r}$, and $\boldsymbol{j}$ represents the current density,

$$
\boldsymbol{j}=\frac{i}{2}\left[\Psi^{*}(\boldsymbol{r}) \nabla \Psi(\boldsymbol{r})-\Psi(\boldsymbol{r}) \nabla \Psi^{*}(\boldsymbol{r})\right] .
$$

\section{TRAJECTORIES IN COMBINED COULOMB AND ELECTRIC FIELDS}

\section{A. Hamiltonian in scaled variables}

In cylindrical coordinates $(\rho, z, \varphi)$, the Hamiltonian of hydrogen atoms in an external electric field $\mathscr{F}$ is

$$
H=\frac{1}{2}\left(p_{\rho}^{2}+p_{z}^{2}+\frac{\ell_{z}^{2}}{\rho^{2}}\right)-\frac{1}{\sqrt{\rho^{2}+z^{2}}}+\mathscr{F} z,
$$

where atomic units, in which $\mathscr{F}$ is measured in $F_{0}=$ $m^{2} e^{5} / \hbar^{4} \approx 5.142 \times 10^{9} \mathrm{~V} / \mathrm{cm}$, are adopted, $p_{\rho}, p_{z}$ denote electron momenta, and $\ell_{z}$ is the $z$ component of angular momenta. Scaled variables are defined by

$$
\begin{gathered}
\widehat{\boldsymbol{r}}=\boldsymbol{r} \mathscr{F}^{1 / 2}, \\
\widehat{\boldsymbol{p}}=\boldsymbol{p} / \mathscr{F}^{1 / 4}, \\
\widehat{t}=t \mathscr{F}^{3 / 4} .
\end{gathered}
$$

It follows that the scaled classical action is

$$
\widehat{S}=S \mathscr{F}^{1 / 4}
$$

and the converted Hamiltonian is of the form,

$$
\widehat{H}=H / \mathscr{F}^{1 / 2}=\frac{1}{2}\left(\widehat{p}_{\rho}^{2}+\widehat{p}_{z}^{2}+\frac{\mathscr{L}_{z}^{2}}{\widehat{\rho}^{2}}\right)-\frac{1}{\sqrt{\widehat{\rho}^{2}+\widehat{z}^{2}}}+\widehat{z},
$$

where $\mathscr{L}_{z}=\ell_{z} \mathscr{F}^{1 / 4}=m \hbar \mathscr{F}^{1 / 4}$. Let us define semiparabolic coordinates using the scaled variables $\widehat{r}, \widehat{z}$ :

$$
\begin{aligned}
u & =\sqrt{\widehat{r}+\widehat{z}}, \\
v & =\sqrt{\widehat{r}-\widehat{z}} .
\end{aligned}
$$

We introduce an independent variable $\tau$ replacing the scaled time variable $\widehat{t}$,

$$
\frac{d \widehat{t}}{d \tau}=u^{2}+v^{2}
$$

and take

$$
\begin{aligned}
\mathscr{H}= & \left(u^{2}+v^{2}\right)(\widehat{H}-\varepsilon)=\frac{1}{2}\left(p_{u}^{2}+p_{v}^{2}\right) \\
& +\frac{\mathscr{L}_{z}^{2}}{2 u^{2}}+\frac{\mathscr{L}_{z}^{2}}{2 v^{2}}-2+\frac{1}{2}\left(u^{4}-v^{4}\right)-\varepsilon\left(u^{2}+v^{2}\right),
\end{aligned}
$$

where $\varepsilon=E / \mathscr{F}^{1 / 2}$ and $\mathscr{H}$ is called the effective Hamiltonian. It is readily proved that the canonical form of the equations of motion and the effective Hamiltonian are conserved. The initial conditions of the equation of motion are

$$
\begin{gathered}
u(0)=0, \\
v(0)=0, \\
p_{u}(0)=2 \cos \left(\theta_{i} / 2\right), \\
p_{v}(0)=2 \sin \left(\theta_{i} / 2\right),
\end{gathered}
$$

where $\theta_{i}$ is the emission angle of the ejected electron.

\section{B. Open orbits from the source to a detector}

As stated earlier, for $\mathscr{L}_{z}=0$, there is a critical angle (derived subsequently) such that trajectories launched from the nucleus with $\theta_{i}=\theta_{c}$ approach an unstable periodic orbit (UPO). This critical orbit is a member of the stable manifold of the UPO, and that stable manifold is a separatrix. All trajectories launched from $0 \leqslant \theta_{i}<\theta_{c}$ stay forever in the vicinity of the nucleus, while those launched with $\theta_{c}<\theta_{i} \leqslant \pi$ escape from the Coulomb well and fall to the detector (Fig. 2). We set the detector at a particular value $z=-\left|z_{\text {det }}\right|$, and integrate the equations of motion with initial conditions, Eqs. (21)-(24). When the trajectories reach the detector, we record the final value of the cylindrical coordinate $\rho_{f}\left(\theta_{i}\right)$. A graph of the final position at the detector, $\rho_{f}$ versus $\log _{10}\left(\theta_{i}-\right.$ $\theta_{c}$ ) is shown in Fig. 3. For computational convenience, we allow $\rho_{f}$ to alternate between negative and positive. From this figure, it is seen that as $\theta_{i}$ approaches $\theta_{c}, \rho_{f}\left(\theta_{i}\right)$ has an infinite number of oscillations because the trajectories are approaching the stable manifold of the UPO.

To compute a semiclassical wave function at each point $\left|\rho_{f}\right|$, we must identify the trajectories that go through that point [i.e., from the single-valued function $\rho_{f}\left(\theta_{i}\right)$ ] we must obtain the inverse function $\theta_{i}\left(\rho_{f}\right)$, which is obviously multivalued, and has an infinite number of branches. For this purpose, we 


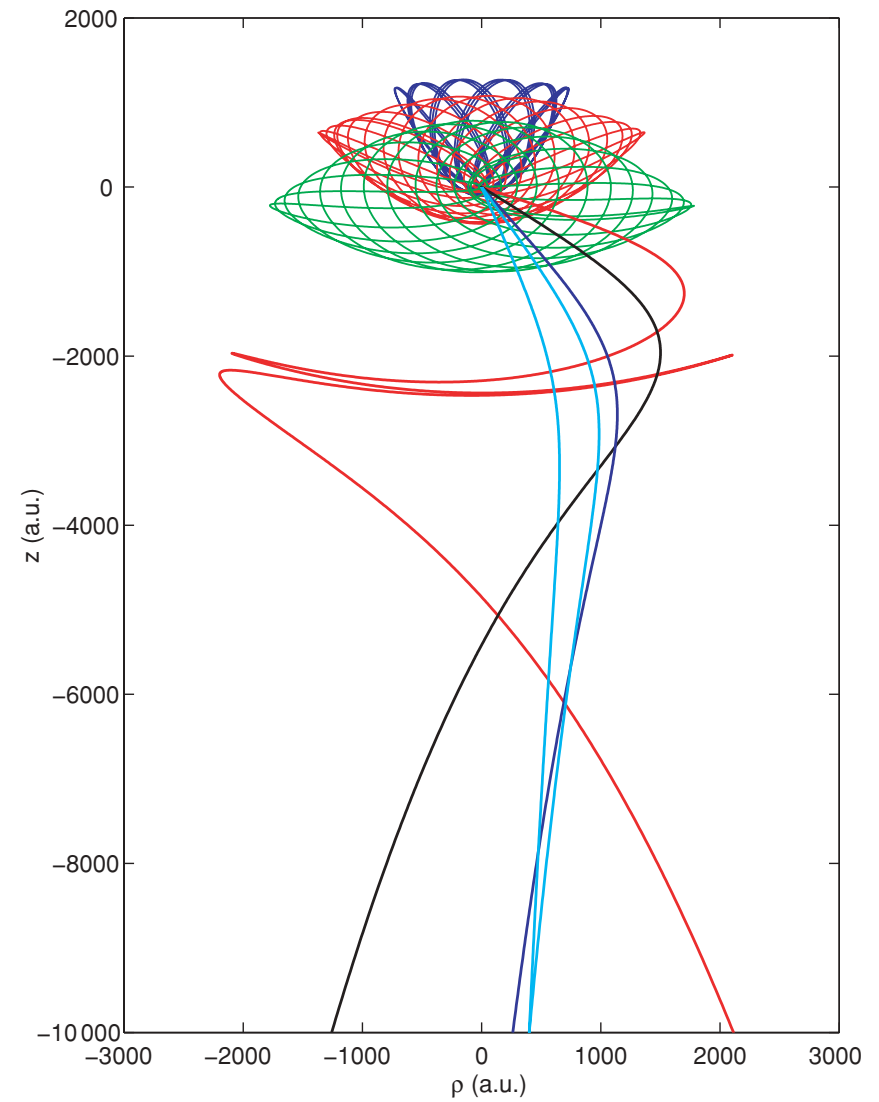

FIG. 2. (Color online) Trajectories representing electron motion in combined Coulomb and electric fields. $\mathrm{H}$ atoms in an electric field with strength $\mathscr{F}=615 \mathrm{~V} / \mathrm{cm}$ are ionized by a laser beam, and the energy of the produced outgoing electrons is $E=-128 \mathrm{~cm}^{-1}$. Three of the eight trajectories, which are restricted to a small region, are bound orbits, while the other five trajectories are open orbits. Two of the five open orbits cross at the same point $|z|=10000$ a.u. and $\rho=400$ a.u. Electrons following bound orbits never reach detectors.

divide the curve in Fig. 3 into segments, with each segment connecting a zero to an extremum.

The emission angles $\theta_{i}^{0, k}$, where $\rho_{f}\left(\theta_{i}^{0, k}\right)=0$, were obtained by taking small steps in $\theta_{i}$, starting with $\theta_{i}=\pi$; whenever $\rho_{f}$ changed sign, the point $\theta_{i}^{0, k}$ could be found numerically by integrating additional trajectories and by using

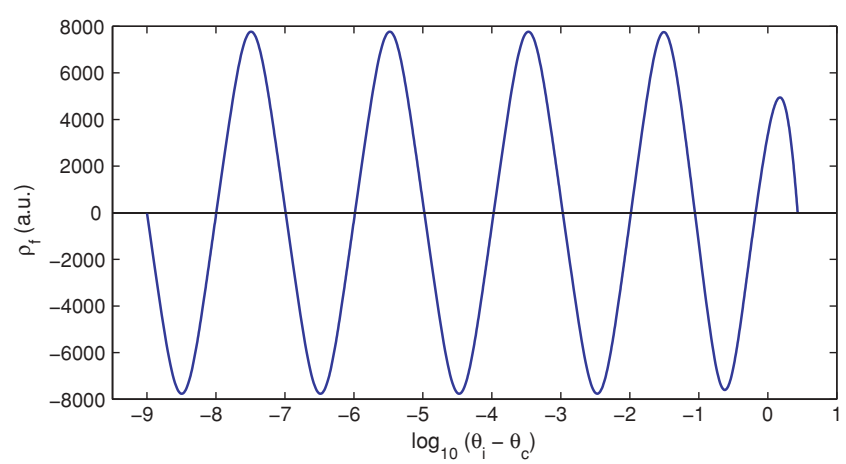

FIG. 3. (Color online) $\rho_{f}$ as a function of $\log _{10}\left(\theta_{i}-\theta_{c}\right)$ with $E=-96 \mathrm{~cm}^{-1}$ and $\mathscr{F}=5914 \mathrm{~V} / \mathrm{cm}$. The detector is located at $z_{\mathrm{det}}=-1 \mu \mathrm{m}$. a Newton root-finding algorithm. Similarly, the values $\theta_{i}^{\mathrm{x}, k}$, where $\rho_{f}\left(\theta_{i}^{\mathrm{x}, k}\right)$ is an extremum, were found by seeking trajectories having the values $\frac{d \rho_{f}}{d \theta_{i}}$ equal to zero. These emission angles $\theta_{i}^{0, k}$ and $\theta_{i}^{\mathrm{x}, k}$ identify starting and ending points of each branch of the inverse function $\theta_{i}(\rho)$. Finally, for each position on the detector at which we wish to obtain semiclassical wave functions, we numerically find each emission angle $\theta_{i}(\rho)$ by integration of additional trajectories with a Newton root-finding algorithm. In principle, an infinite number of trajectories arrive at each point in the classically allowed region of the detector. In practice, the amplitudes associated with these trajectories decrease quickly, and only a few are needed for an accurate calculation.

\section{Computation of actions, Jacobians, and Maslov indices}

The classical actions $S$ are evaluated by numerically integrating an additional equation,

$$
\frac{d \widehat{S}}{d \tau}=p_{u}^{2}+p_{v}^{2},
$$

together with the equations of motion. However, such a computation is suitable only within a moderately large distance. When the electron wave propagates up to a macroscopic size, the velocities of the electron and relevant actions become larger and larger, and, therefore, Eq. (25) may no longer provide the actions with sufficient accuracy. One has to seek action differences, rather than the actions themselves to obtain reliable phases. We use $\Delta S_{i}\left(\rho, z_{\text {det }}\right)=S_{i}\left(\rho, z_{\text {det }}\right)-S_{\pi}\left(0, z_{\text {det }}\right)$ to replace $S_{i}(\mathbf{r})$ in Eq. (4), where $S_{\pi}$ represents the action on the trajectory with the initial emission angle $\theta_{i}=\pi$, and we use an analytical formula, Eq. (29), to compute the difference of the scaled actions $\Delta \widehat{S}_{i}$ associated with motion in the $v$ coordinate. It is well known that the equations of motion for the Hamiltonian Eq. (20) are separable in the $u$ and $v$ coordinates,

$$
\begin{gathered}
\frac{1}{2} p_{u}^{2}+\frac{1}{2} u^{4}-\varepsilon u^{2}+\frac{\mathscr{L}_{z}^{2}}{2 u^{2}}-1=\beta, \\
\frac{1}{2} p_{v}^{2}-\frac{1}{2} v^{4}-\varepsilon v^{2}+\frac{\mathscr{L}_{z}^{2}}{2 v^{2}}-1=-\beta,
\end{gathered}
$$

with

$$
\beta=\cos \theta_{i}+\frac{1}{2} \widehat{r}_{i}^{2} \sin ^{2} \theta_{i}-\frac{\mathscr{L}_{z}^{2} \cos \theta_{i}}{\widehat{r}_{i} \sin ^{2} \theta_{i}},
$$

where $\beta$ is a conserved quantity [16], and its initial value is $\cos \theta_{i}$ if $\mathscr{L}_{z}=0$. The action difference associated with motion in the $v$ coordinate is calculated using Eq. (27):

$$
\begin{aligned}
\Delta \widehat{S}_{i}^{v}\left(\rho_{f}, z_{\mathrm{det}}\right)= & \int_{0}^{v_{f}}\left(\sqrt{v^{4}+2 \varepsilon v^{2}-\frac{\mathscr{L}_{z}^{2}}{v^{2}}+2-2 \beta}\right. \\
& -\sqrt{\left.v^{4}+2 \varepsilon v^{2}-\frac{\mathscr{L}_{z}^{2}}{v^{2}}+4\right) d v}
\end{aligned}
$$

For the classical amplitude $A_{j}(\mathbf{r})$, one needs the threedimensional Jacobian $J(t, \mathbf{r})$, which can be further expressed 
in terms of the two-dimensional Jacobian $J_{2}(t, \theta)$,

$$
\begin{aligned}
J(t, \mathbf{r}) & =\frac{\partial(x, y, z)}{\partial(t, \theta, \varphi)}=\frac{\partial(x, y, z) \partial(\rho, z, \varphi) \partial(u, v, \varphi) \partial(\tau, \theta, \varphi)}{\partial(\rho, z, \varphi) \partial(u, v, \varphi) \partial(\tau, \theta, \varphi) \partial(t, \theta, \varphi)} \\
& =u v J_{2}(\tau, \theta),
\end{aligned}
$$

with

$$
J_{2}(\tau, \theta)=\frac{\partial(u, v)}{\partial(\tau, \theta)}=\left(\begin{array}{ll}
\left.\frac{\partial u}{\partial \tau}\right|_{\theta} & \left.\frac{\partial u}{\partial \theta}\right|_{\tau} \\
\left.\frac{\partial v}{\partial \tau}\right|_{\theta} & \left.\frac{\partial v}{\partial \theta}\right|_{\tau}
\end{array}\right) .
$$

For convenience of numerical calculations, we give four additional equations obtained by differentiating the equations of motion with respect to $\theta$,

$$
\begin{gathered}
\frac{d}{d \tau}\left(\frac{\partial u}{\partial \theta}\right)=\frac{\partial p_{u}}{\partial \theta}, \\
\frac{d}{d \tau}\left(\frac{\partial v}{\partial \theta}\right)=\frac{\partial p_{v}}{\partial \theta}, \\
\frac{d}{d \tau}\left(\frac{\partial p_{u}}{\partial \theta}\right)=\left(-3 \frac{\mathscr{L}_{z}^{2}}{u^{3}}+2 \varepsilon-6 u^{2}\right) \frac{\partial u}{\partial \theta}, \\
\frac{d}{d \tau}\left(\frac{\partial p_{v}}{\partial \theta}\right)=\left(-3 \frac{\mathscr{L}_{z}^{2}}{v^{3}}+2 \varepsilon+6 v^{2}\right) \frac{\partial v}{\partial \theta}
\end{gathered}
$$

They are solved together with the equations of motion. The final values of the Jacobians are obtained when the electron hits the detector. The initial values of the four unknown quantities in Eqs. (32)-(35) may be easily obtained from Eqs. (21)-(24). The Maslov index increases by 1 every time that $u, v$, or $J_{2}$ passes through zero.

An alternative formula for $J_{2}(\tau, \theta)$, when the electron reaches the detector, is

$$
J_{2}(\tau, \theta)=\frac{u p_{u}-v p_{v}}{u^{2}+v^{2}}\left(\frac{\partial \widehat{\rho}_{f}}{\partial \theta}\right)_{z},
$$

A derivative is given in Appendix.

\section{Bound orbits corresponding to quantum tunneling}

From Eqs. (26) and (27), effective potentials of $\mathrm{H}$ atoms in an electric field in the $u$ and $v$ coordinates are written in the form

$$
\begin{gathered}
U(u)=\frac{1}{2} u^{4}-\varepsilon u^{2}+\frac{\mathscr{L}_{z}^{2}}{2 u^{2}}, \\
V(v)=-\frac{1}{2} v^{4}-\varepsilon v^{2}+\frac{\mathscr{L}_{z}^{2}}{2 v^{2}},
\end{gathered}
$$

and corresponding effective energies for the Hamiltonian are $1+\beta$ and $1-\beta$, respectively. If we let $\mathscr{L}_{z}$ be zero, $V(v)$ displays a potential barrier, with a maximum

$$
V_{\max }(v)=\frac{1}{2} \varepsilon^{2}=1-\cos \theta_{c},
$$

at $v=\sqrt{-\varepsilon}$. From the previous equation, one can show that the critical angle is

$$
\theta_{c}=2 \sin ^{-1}\left(\frac{-E}{2 \sqrt{\mathscr{F}}}\right) .
$$

As an example, we plot $U(u)$ and $V(v)$ in Fig. 4. The electron is trapped forever by the potential barrier, according to classical theory, if the effective energy is smaller than $1-\cos \theta_{c}$, or

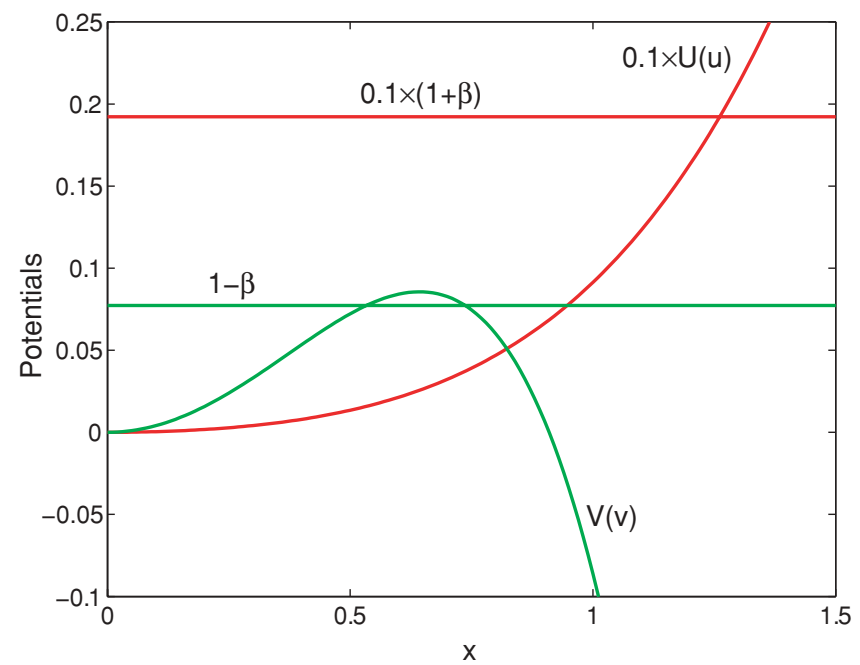

FIG. 4. (Color online) Effective potentials of $\mathrm{H}$ atoms in electric fields with strength $\mathscr{F}=5714 \mathrm{~V} / \mathrm{cm}$ in the $u$ and $v$ coordinates. $\mathrm{X}$ denotes $u$ or $v$. The angular momentum $\mathscr{L}_{z}$ is taken to be zero, and electron energy $E=-95.733 \mathrm{~cm}^{-1}$, where a resonance is located (see Table I). The horizontal lines indicate effective energies for the $u$ and $v$ motions, $1+\beta$ and $1-\beta$, respectively. For $v$ motion, the effective potential has a barrier.

in other words, if the emission angle is smaller than the critical angle $\theta_{c}$, as shown in Fig. 2. However, quantum waves corresponding to some trajectories with special emission angles smaller than $\theta_{c}$ may tunnel through the potential barrier. Such trajectories correspond to quasibound resonances having energies and emission angles determined by the BohrSommerfeld quantization conditions,

$$
\begin{aligned}
& \frac{1}{\mathscr{F}^{1 / 4}} \int_{0}^{u_{\mathrm{ctp}}} p_{u} d u=\left(n_{u}+\frac{1}{2}\right) \pi, \\
& \frac{1}{\mathscr{F}^{1 / 4}} \int_{0}^{v_{\mathrm{ctp}}} p_{v} d v=\left(n_{v}+\frac{1}{2}\right) \pi,
\end{aligned}
$$

where $u_{\text {ctp }}$ and $v_{\text {ctp }}$ denote classical turning points of $U(u)$ and $V(v)$, respectively, and $n_{u}$ and $n_{v}$ are quantum numbers. As shown in Fig. 4, the intersections of the potentials and their corresponding horizontal lines determine classical turning points. For given $n_{u}$ and $n_{v}$, one may solve the preceding equations to find $E$ and $\beta$.

In Fig. 5, we plot contour lines of $n_{u}(E, \beta)$ and $n_{v}(E, \beta)$ at an electric field $\mathscr{F}=5714 \mathrm{~V} / \mathrm{cm}$. The intersection points of the $n_{u}$ and $n_{v}$ lines correspond to resonance states. Table I lists several computed resonance positions at $\mathscr{F}=5714 \mathrm{~V} / \mathrm{cm}$, obtained by solutions of Eqs. (41) and (42). Comparison is made with quantum-mechanical calculations $[17,18]$ and shows good agreement. Later, we will see how these resonance states tunneling through the potential barrier affect interference patterns on a detector.

\section{CORRECTING SINGULARITIES}

The semiclassical approximation to the wave function diverges whenever the Jacobian $J\left(t_{f}, \boldsymbol{q}\right)$ passes through zero. In the current case, this happens whenever the cylindrical coordinate $\rho$ goes to zero or passes through an extremum. 


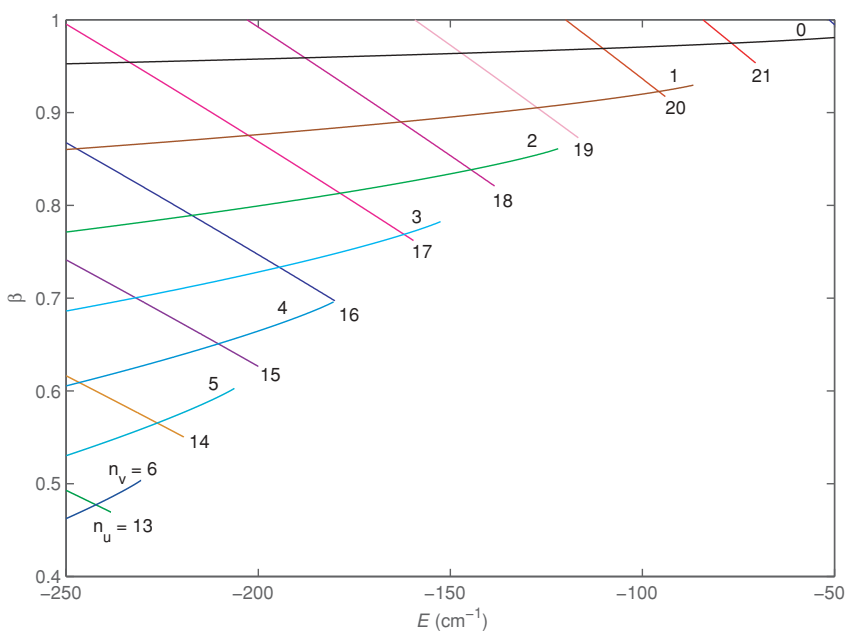

FIG. 5. (Color online) Contour plots of $n_{u}(E, \beta)$ and $n_{v}(E, \beta)$ of $\mathrm{H}$ atoms in electric fields $\mathscr{F}=5714 \mathrm{~V} / \mathrm{cm}$. The angular momentum is taken to be zero (i.e., $m=0$ ). The given contour lines are $n_{u}=$ $13,14, \ldots, 22$ and $n_{v}=0,1, \ldots, 6$. Any one of the intersection points of the $n_{u}$ and $n_{v}$ lines corresponds to a resonance state, specified by $\left(n_{u}, n_{v}, m\right)$. For example, $(17,0,0)$ is located at the intersection of $n_{u}=17$ and $n_{v}=0$ with $E=-233.507 \mathrm{~cm}^{-1}$.

To fix the divergent behaviors, approximations have been introduced (see Ref. [19] and reference therein), and various kinds of caustic surfaces are classified in catastrophe theory to deal with different generic cases, such as folds or cusps. In our system, which is three dimensional, but reducible to two dimensions due to the cylindrical symmetry, the extrema in $\rho$ correspond to fold caustics, while singularities at $\rho=0$ are nongeneric cylindrical foci.

\section{A. Caustics}

It has been shown [19] that electron waves undergoing refraction near a fold-type caustic surface resemble Airy functions (see Ref. [20] for properties of Airy functions), and the uniform Airy approximation describes the refracting electron waves near this type of caustic surface very well. The theoretical details of the uniform Airy approximation have been discussed in Ref. [19], and here we only present the formulas we use in this paper.

TABLE I. Comparison of resonance positions (in $\mathrm{cm}^{-1}$ ) of $\mathrm{H}$ atoms in electric fields with $\mathscr{F}=5714 \mathrm{~V} / \mathrm{cm}$ between the current and other calculations.

\begin{tabular}{lrrr}
\hline \hline$\left(n_{u}, n_{v}, m\right)$ & This work & Ref. [17] & Ref. [18] \\
\hline$(22,0,0)$ & -46.406 & -46.925 & -46.53 \\
$(21,0,0)$ & -76.798 & -77.176 & -76.77 \\
$(20,0,0)$ & -110.245 & & -110.13 \\
$(20,1,0)$ & -95.733 & -96.196 & -95.87 \\
$(19,1,0)$ & -127.377 & -127.647 & -127.31 \\
$(18,1,0)$ & -162.873 & & -162.74 \\
$(18,2,0)$ & -144.625 & -144.915 & -144.64 \\
$(17,1,0)$ & -202.566 & & -202.40 \\
$(17,2,0)$ & -178.699 & & -178.59 \\
$(17,3,0)$ & -162.059 & -162.413 & -162.21 \\
\hline \hline
\end{tabular}

Referring to Fig. 2, we see that for $\theta_{i}$ between $\theta_{i}^{0, k}$ and $\theta_{i}^{0, k+1}$, there are two semiclassical terms that contribute to the wave function at the detector. The uniform Airy approximation corrects and combines this pair of semiclassical terms. We label these two terms $a$ and $b . a$ is the one with larger $\theta_{i}$, and it has the smaller action of the pair. The two actions $S_{a}$ and $S_{b}$ become equal at $\theta_{i}^{\mathrm{x}, k}$, where $\rho$ has an extremum. We define

$$
\begin{gathered}
\mathcal{W}_{k}(\mathbf{r})=\left(S_{a}+S_{b}\right) / 2, \\
\zeta_{k}(\mathbf{r})=\left[3\left(S_{b}-S_{a}\right) / 4\right]^{2 / 3}, \\
\mathcal{A}_{k}^{ \pm}(\boldsymbol{r})=\psi_{\text {out }}^{a}\left(\boldsymbol{r}_{0}\right) A_{a}(\mathbf{r}) \pm \psi_{\text {out }}^{b}\left(\boldsymbol{r}_{0}\right) A_{b}(\boldsymbol{r}) .
\end{gathered}
$$

In the uniform approximation, the wave function is expressed as

$$
\Psi_{\mathrm{uni}}^{(k)}(\boldsymbol{r})=\gamma_{k}(\boldsymbol{r}) \mathrm{Ai}\left(-\zeta_{k}(\boldsymbol{r})\right)-i \delta_{k}(\boldsymbol{r}) \mathrm{Ai}^{\prime}\left(-\zeta_{k}(\boldsymbol{r})\right),
$$

with

$$
\begin{gathered}
\gamma_{k}(\boldsymbol{r})=(-1)^{k} \sqrt{\pi} e^{i \mathcal{W}_{k}(r)} \mathcal{A}_{k}^{+}(\boldsymbol{r}) \zeta_{k}(\boldsymbol{r})^{1 / 4}, \\
\delta_{k}(\boldsymbol{r})=(-1)^{k} \sqrt{\pi} e^{i \mathcal{W}_{k}(r)} \mathcal{A}_{k}^{-}(\boldsymbol{r}) \zeta_{k}(\boldsymbol{r})^{-1 / 4},
\end{gathered}
$$

where $\mathrm{Ai}(x)$ and $\mathrm{Ai}^{\prime}(x)$ represent the Airy function and its derivative, respectively.

Let $\rho_{f}^{\mathrm{x}, k}$ represent the $k$ th extremum of $\rho_{f}$. In order to calculate the wave functions in the classically forbidden region $\left|\rho_{f}\right|>\left|\rho_{f}^{\mathrm{x}, k}\right|$, we performed an analytical continuation of physical quantities in the previous equations. In the classically allowed region, we fitted $\zeta_{k}$ to a linear function passing through zero at $\left|\rho_{f}\right|=\left|\rho_{f}^{\mathrm{x}, k}\right|$ [i.e., $S_{b}-S_{a}$ is proportional to $\left.\left(\left|\rho_{f}\right|-\left|\rho_{f}^{\mathrm{x}, k}\right|\right)^{3 / 2}\right]$. Also in the classically allowed region, we fitted $\mathcal{W}_{k}$ and $\mathcal{A}_{k}^{ \pm} \zeta^{ \pm 1 / 4}$ to quadratic polynomials of $\left|\rho_{f}\right|-\left|\rho_{f}^{\mathrm{x}, k}\right|$. These fitting functions were then extrapolated into the classically forbidden regions. Finally, we sum $\Psi_{\text {uni }}^{(k)}(\boldsymbol{r})$ over all the $\theta_{i}$ intervals to obtain total wave functions in the uniform Airy approximation.

\section{B. Cylindrical foci}

The Schrödinger equation of $\mathrm{H}$ atoms in an electric field is separable in the $\rho$ and $z$ coordinates if $\rho$ is small and $z$ is sufficiently large. Its solution for $\ell_{z}=0$ in the $\rho$ coordinate is the Bessel function [20] of zero order $J_{0}\left(p_{\rho} \rho\right)$.

Let us identify the $k$ th point where $\rho_{f}=0$ in Fig. 3. The point corresponds to the emission angle $\theta_{i}^{0, k}$. We define $S^{0, k}$ as the action from the atom to the detector along the trajectory with the emission angle $\theta_{i}^{0, k}$, and record the $\rho$ component of the momentum at the detector $p_{\rho}^{0, k}$. Near $\rho=0$, we have

$$
S_{ \pm}=S^{0, k} \pm\left|p_{\rho}^{0, k}\right| \rho .
$$

The Jacobian there is

$$
J\left(\rho, z_{\mathrm{det}}\right)=\mathscr{J}^{0, k} \rho,
$$

where $\mathscr{J}^{0, k}$ is a constant. For a given $\rho$, the semiclassical wave function for the two terms near the $k$ th point with $k \geqslant 2$ is

$$
\begin{aligned}
\Psi^{k}\left(\rho, z_{\mathrm{det}}\right)= & \psi_{\text {out }}\left(\theta_{i}^{0, k}, r_{0}\right)\left|\frac{J\left(\theta_{i}^{0, k}, r_{0}\right)}{J\left(\rho, z_{\mathrm{det}}\right)}\right|^{1 / 2} \\
& \times e^{-i \mu_{-} \pi / 2}\left[e^{i S_{-}}+e^{i\left(S_{+}-\pi / 2\right)}\right],
\end{aligned}
$$



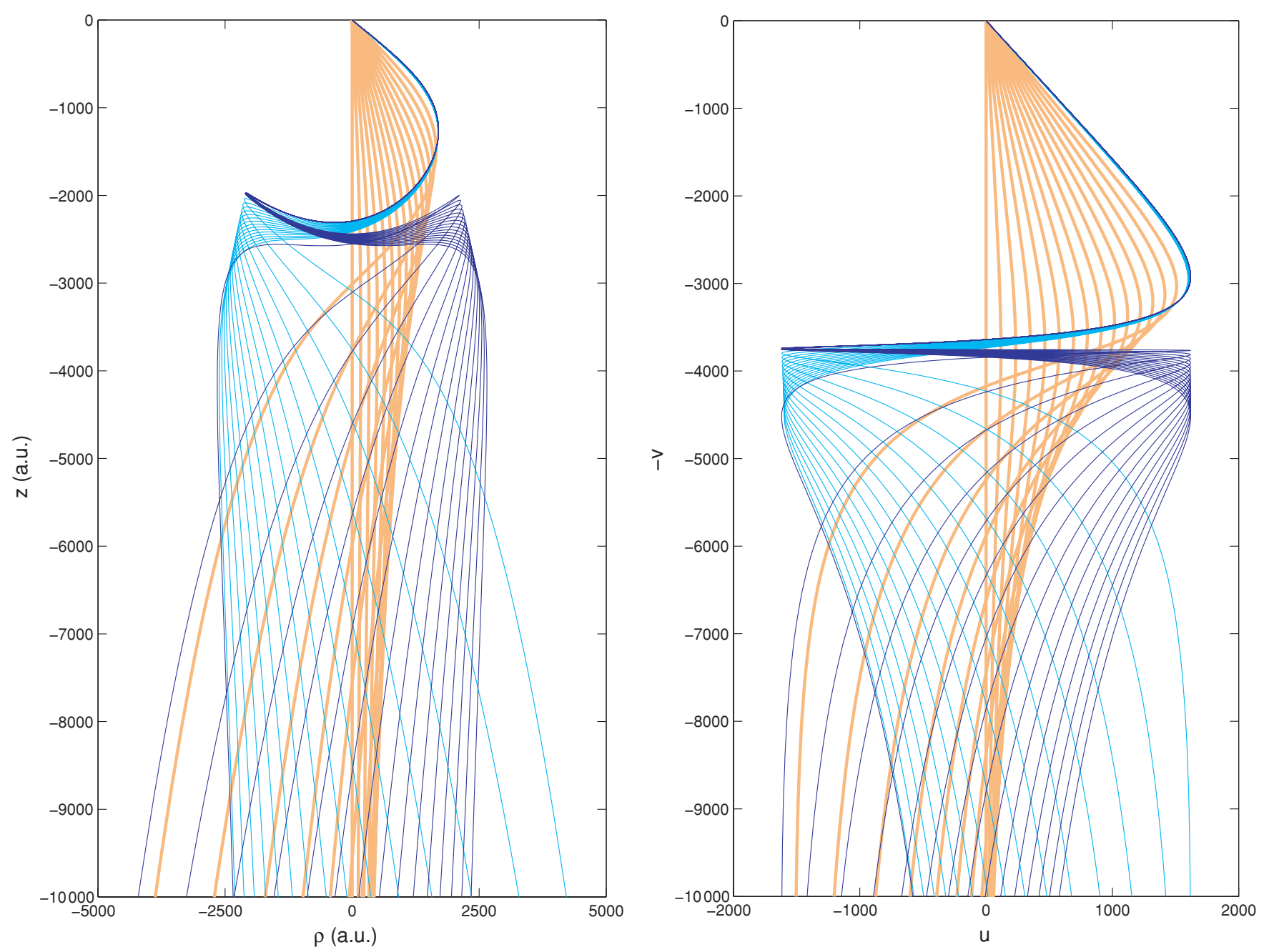

FIG. 6. (Color online) Motion of electrons ejected from $\mathrm{H}$ atoms in an electric field $\mathscr{F}=615 \mathrm{~V} / \mathrm{cm}$. The electron energy is $E=-128 \mathrm{~cm}^{-1}$. Trajectories are drawn in both cylindrical (left panel) and semiparabolic (right panel) coordinates. The position of the detector is taken to be $-30 \mu \mathrm{m}$ away from the atomic source, and each color, orange (wide light gray), cyan (narrow light gray), or blue (dark gray) represents one range of emission angles from $\theta_{i}^{0, k}$ to $\theta_{i}^{0, k+1}$.

where $\mu_{-}$represents the Maslov index corresponding to $S_{-}$. Substituting Eqs. (49) and (50) into Eq. (51) yields

$$
\begin{aligned}
\Psi^{k}\left(\rho, z_{\mathrm{det}}\right)= & \psi_{\text {out }}\left(\theta_{i}^{0, k}, r_{0}\right)\left|\frac{J\left(\theta_{i}^{0, k}, r_{0}\right)}{\mathscr{J}^{0, k} \rho}\right|^{1 / 2} \\
& \times e^{i\left(S^{0, k}-\mu_{-} \pi / 2\right)}\left[e^{-i\left|p_{\rho}^{0, k}\right| \rho}+e^{i\left(\left|p_{\rho}^{0, k}\right| \rho-\pi / 2\right)}\right]
\end{aligned}
$$

This must correspond to a constant times the Bessel function $\mathscr{D}_{k} J_{0}\left(p_{\rho}^{0, k} \rho\right)$. Comparing the asymptotic form of the Bessel function with Eq. (52), the constant $\mathscr{D}_{k}$ is determined to be

$$
\begin{aligned}
\mathscr{D}_{k}= & \sqrt{2 \pi} e^{-i \pi / 4}\left|p_{\rho}^{0, k}\right|^{1 / 2} \psi_{\text {out }}\left(\theta_{i}^{0, k}, r_{0}\right)\left|\frac{J\left(\theta_{i}^{0, k}, r_{0}\right)}{\mathscr{J}^{0, k}}\right|^{1 / 2} \\
& \times e^{i\left(S^{0, k}-\mu_{-} / 2\right)}
\end{aligned}
$$

By summing all $k$, one finally obtains the repaired wave functions near $\rho=0$,

$$
\Psi\left(\rho, z_{\mathrm{det}}\right)=\Psi^{1}\left(\rho, z_{\mathrm{det}}\right)+\sum_{k=2}^{\infty} \mathscr{D}_{k} J_{0}\left(p_{\rho}^{0, k} \rho\right),
$$

where $\Psi^{1}\left(\rho, z_{\text {det }}\right)$ represents the nondivergent semiclassical wave function for emission angles near $\theta_{i}^{0,1}=\pi$.

\section{COMPUTATIONAL RESULTS AND DISCUSSION}

It has been illustrated in Fig. 2 that electron waves along open orbits may propagate to a large distance. To show how the combined Coulomb and uniform electric fields shape electron spatial distributions, we plot classical trajectories of the ejected photoelectron at $E=-128 \mathrm{~cm}^{-1}$ and $\mathscr{F}=$ $615 \mathrm{~V} / \mathrm{cm}$ in cylindrical and semiparabolic coordinates in Fig. 6. The open orbits in every region with initial emission angles from $\theta_{i}^{0, k}$ to $\theta_{i}^{0, k+1}$ are represented by single color curves. For the current energy and electric field, these angles are $\theta_{i}^{0, k}=180^{\circ}, 115.97531^{\circ}, 114.97623^{\circ}, 114.96038^{\circ}$. (Note 


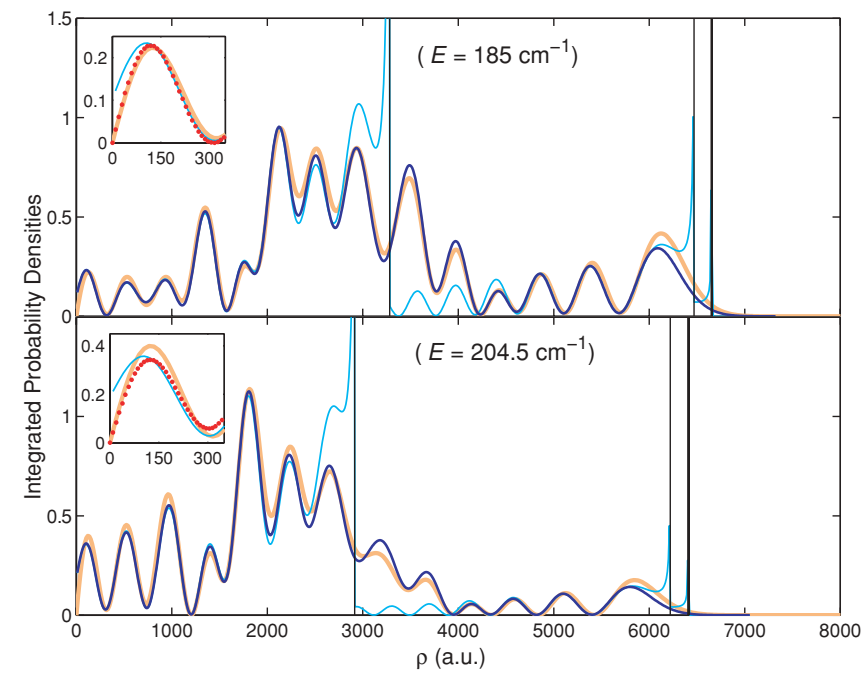

FIG. 7. (Color online) Probability densities, integrated over the angular variable $\varphi$ of cylindrical coordinates $(\rho, z, \varphi)$, of the ejected electron at two energies $E=-185 \mathrm{~cm}^{-1}$ and $-204.5 \mathrm{~cm}^{-1}$. $\mathrm{H}$ atoms are placed in an electric field $\mathscr{F}=5714 \mathrm{~V} / \mathrm{cm}$, and the detector is located at $z_{\mathrm{det}}=-1 \mu \mathrm{m}$ from the atoms. The cyan (narrow light gray) curves represent the results from the semiclassical approximation, which diverges at caustics denoted with black vertical lines, and the blue (dark gray) curves denote those from the uniform approximation. Caustics near $\rho_{f}=7680 a_{0}$ are too close to be distinguishable. The orange (wide light gray) curves are from quantum-mechanical calculations. The solid dots near $\rho=0$ a.u. represent the Besselfunction approximation.

that the value of $\theta_{i}^{0, k}$ depends on the position of the detector as well as the scaled energy $\varepsilon=E / \mathscr{F}^{1 / 2}$.) The behavior of $\rho_{f}$ versus $\theta_{i}$ is qualitatively similar to that shown in Fig. 3.

Figure 7 plots probability density distributions, integrated over the angular variable $\varphi$ of cylindrical coordinates $(\rho, z, \varphi)$, of the ejected photoelectron at two energies $E=-185 \mathrm{~cm}^{-1}$ and $-204.5 \mathrm{~cm}^{-1}$, calculated in the semiclassical and uniform approximations, and in the fully quantum-mechanical method (see the accompanying paper for theoretical details). In the present calculations, we assume that ground-state $\mathrm{H}$ atoms placed in an electric field $\mathscr{F}=5714 \mathrm{~V} / \mathrm{cm}$ are first excited to the state $\frac{1}{\sqrt{2}}\left(\psi_{2 s}-\psi_{2 p}\right)$ in the $n=2$ shell, and then ionized by a $\pi$-polarized laser. This initial state and a $\pi$-polarized laser are always assumed in all later calculations. The detector is located at $z_{\operatorname{det}}=-1 \mu \mathrm{m}$. Semiclassical divergences at caustics are visible, and such divergences were repaired by means of the uniform approximation. We also fixed the divergent behaviors of the wave functions near $\rho=0$ a.u. using the Bessel functions. It is seen that semiclassical and fully quantum-mechanical calculations are in good agreement. This is because these two energies are not close to any resonance. Later, we will show that quantum tunneling effects dominate the interference patterns if the electron energy is close to a resonance.

The change of spatial electron current distributions with distances $z_{\text {det }}$ from $\mathrm{H}$ atomic resources is investigated. We took the electric field to be $\mathscr{F}=5714 \mathrm{~V} / \mathrm{cm}$ and electron energy $E=-204.5 \mathrm{~cm}^{-1}$. The electron current distributions at $z_{\text {det }}=$ $-100 \mu \mathrm{m},-1000 \mu \mathrm{m},-1 \mathrm{~cm},-10 \mathrm{~cm}$, and $-0.5 \mathrm{~m}$ are

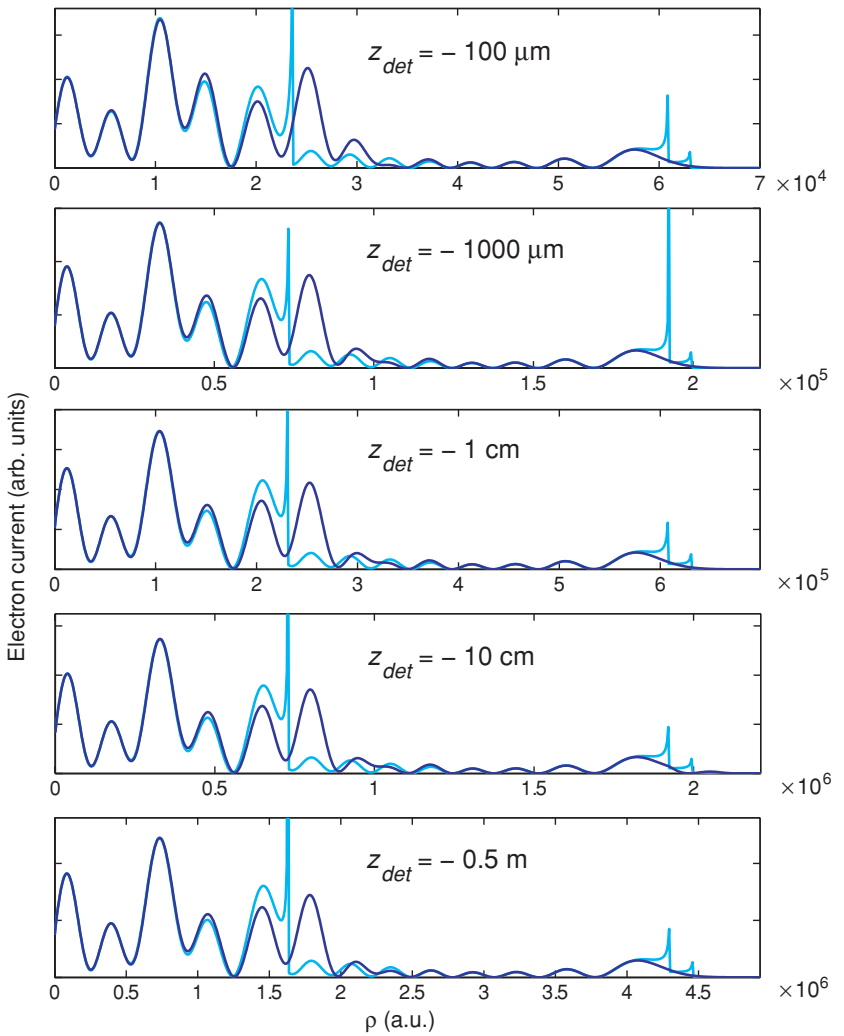

FIG. 8. (Color online) The change of spatial electron current distributions with distances $z_{\text {det }}$ from $\mathrm{H}$ atomic resources. The electric field is $\mathscr{F}=5714 \mathrm{~V} / \mathrm{cm}$ and electron energy is $E=-204.5 \mathrm{~cm}^{-1}$. The cyan (light gray) and blue (dark gray) curves, respectively, represent results from the semiclassical and uniform approximations.

illustrated in Fig. 8. The electron current distributions spread out more widely with increasing $z_{\text {det }}$, but the shapes remain nearly unchanged. A contour plot of electron current distributions corresponding to $z_{\mathrm{det}}=-0.5 \mathrm{~m}$ is drawn in Fig. 9 . It should be pointed out that in all cases we studied, the probability densities and the current densities have the same shapes, and differ only by a constant.

To see effects of resonances on interference patterns, we calculated wave functions of the ejected photoelectron at energy $E=-96 \mathrm{~cm}^{-1}$, a position close to a resonance $\left(n_{u}, n_{v}, m\right)=$ $(20,1,0)$, using semiclassical and uniform approximations, and

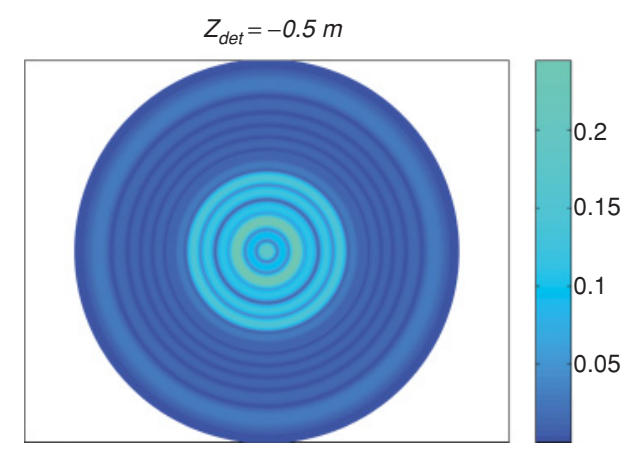

FIG. 9. (Color online) Contour plot of electron current distributions in the uniform approximation corresponding to $z_{\text {det }}=-0.5 \mathrm{~m}$ in Fig. 8. 


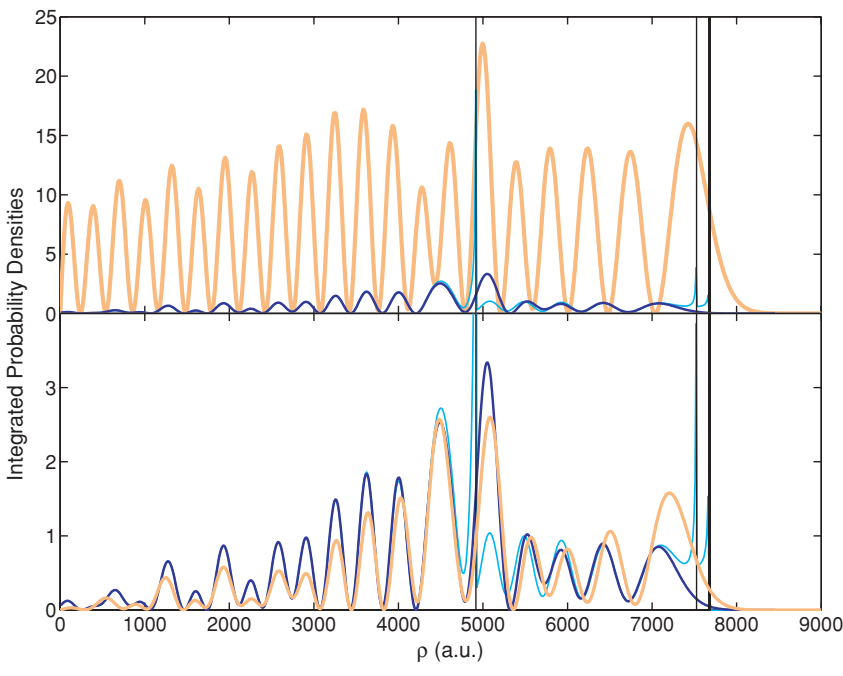

FIG. 10. (Color online) Same as in Fig. 7, but for the ejected electron energy $E=-96 \mathrm{~cm}^{-1}$, near which there is a resonance state $(20,1,0)$. In the lower panel, the contribution of this resonance state [orange (wide light gray)] is removed from the integrated probability densities. One may observe the striking tunneling effect by comparing the upper and lower panels. Note the changes in scale.

the fully quantum-mechanical method. The electric field is taken to be $\mathscr{F}=5714 \mathrm{~V} / \mathrm{cm}$, and the detector is located at $z_{\text {det }}=-1 \mu \mathrm{m}$. Probability densities, integrated over the angular variable $\varphi$ of cylindrical coordinates $(\rho, z, \varphi)$, of the electron are drawn in Fig. 10. The remarkable difference, as seen in the upper panel of Fig. 10, between the semiclassical and quantum-mechanical results is attributed to quantum tunneling from the resonance state $(20,1,0)$ near $E=-96 \mathrm{~cm}^{-1}$. This resonance tunnels through the potential barrier and dominantly contributes to the observed interference. To display this effect plainly, we can remove the contribution of the resonance state from the probability densities using the quantum-mechanical method described in the following paper [21]. The resonance state is contained in one term with $n_{u}=20$ in the summation over $n_{1}$ in Eq. (22) of Ref. [21] (note that $n_{u}$ is denoted as $n_{1}$ therein). By omitting that term, we draw the resultant curve accompanied by those from the semiclassical and uniform approximations in the lower panel of Fig. 10. The agreement between the quantum calculation and the open-orbit theory is significantly improved. Such remarkable tunneling effects should be visible in photoionization microscopy experiments for hydrogen atoms in electric fields.

\section{SUMMARY}

We developed a semiclassical open-orbit theory, based on the assumption that electron waves propagate along classical paths from a pointlike source to a detector. This theory provides a clear and intuitive physical picture to interpret and to predict structures of observable geometrical interference patterns in photoionization microscopy. We calculated electron probability densities and current density distributions of hydrogen atoms in electric fields at several electron energies using open-orbit theory. The results have been found to be in good agreement with quantum-mechanical calculations at electron energies away from resonance states. By using the Bohr-Sommerfeld quantization condition, positions of resonance states can be predicted from semiclassical theory. Resonance positions of $\mathrm{H}$ atoms in electric fields are presented. A remarkable quantum tunneling phenomenon has been found. Such a tunneling effect should be visible in photoionization microscopy measurements on hydrogen atoms.

\section{ACKNOWLEDGMENTS}

One of the authors (L.B.Z.) thanks Mengli Du for his helpful discussion. This work is supported by the National Science Foundation.

\section{APPENDIX: DERIVATIVES OF $\rho_{f}$ WITH RESPECT TO THE EMISSION ANGLES $\theta_{i}$}

To seek the emission angles at the extrema of $\rho_{f}$, one needs calculations of $\left(\frac{\partial \rho_{f}}{\partial \theta}\right)_{z}$. In this appendix, we show how to obtain this derivative. The inverse functions of $u(\widehat{\rho}, \widehat{z})$ and $v(\widehat{\rho}, \widehat{z})$ are

$$
\begin{aligned}
& \widehat{z}=\widehat{z}(u(\tau, \theta), v(\tau, \theta)), \\
& \widehat{\rho}=\widehat{\rho}(u(\tau, \theta), v(\tau, \theta)) .
\end{aligned}
$$

Differentiating Eq. (A1) produces

$$
\begin{aligned}
d \widehat{z}= & \left(\frac{\partial \widehat{z}}{\partial u}\right)_{v}\left[\left(\frac{\partial u}{\partial \tau}\right)_{\theta} d \tau+\left(\frac{\partial u}{\partial \theta}\right)_{\tau} d \theta\right] \\
& +\left(\frac{\partial \widehat{z}}{\partial v}\right)_{u}\left[\left(\frac{\partial v}{\partial \tau}\right)_{\theta} d \tau+\left(\frac{\partial v}{\partial \theta}\right)_{\tau} d \theta\right] .
\end{aligned}
$$

Assuming that $z$ is a constant, we further have

$$
\begin{aligned}
\left(\frac{\partial \tau}{\partial \theta}\right)_{z} & =-\frac{\left(\frac{\partial \widehat{z}}{\partial u}\right)_{v}\left(\frac{\partial u}{\partial \theta}\right)_{\tau}+\left(\frac{\partial \widehat{z}}{\partial v}\right)_{u}\left(\frac{\partial v}{\partial \theta}\right)_{\tau}}{\left(\frac{\partial \widehat{z}}{\partial u}\right)_{v}\left(\frac{\partial u}{\partial \tau}\right)_{\theta}+\left(\frac{\partial \widehat{z}}{\partial v}\right)_{u}\left(\frac{\partial v}{\partial \tau}\right)_{\theta}} \\
& =-\frac{u\left(\frac{\partial u}{\partial \theta}\right)_{\tau}-v\left(\frac{\partial v}{\partial \theta}\right)_{\tau}}{u p_{u}-v p_{v}} .
\end{aligned}
$$

From Eq. (A2), the derivative of $\rho$ with respect to $\theta$ when fixing $z$ is obtained:

$$
\begin{gathered}
\left(\frac{\partial \widehat{\rho}_{f}}{\partial \theta}\right)_{z}=\left(\frac{\partial \widehat{\rho}}{\partial u}\right)_{v}\left[\left(\frac{\partial u}{\partial \tau}\right)_{\theta}\left(\frac{\partial \tau}{\partial \theta}\right)_{z}+\left(\frac{\partial u}{\partial \theta}\right)_{\tau}\right]_{\tau} \\
+\left(\frac{\partial \widehat{\rho}}{\partial v}\right)_{u}\left[\left(\frac{\partial v}{\partial \tau}\right)_{\theta}\left(\frac{\partial \tau}{\partial \theta}\right)_{z}+\left(\frac{\partial v}{\partial \theta}\right)_{\tau}\right] \\
=v\left[p_{u}\left(\frac{\partial \tau}{\partial \theta}\right)_{z}+\left(\frac{\partial u}{\partial \theta}\right)_{\tau}\right]+u\left[p_{v}\left(\frac{\partial \tau}{\partial \theta}\right)_{z}+\left(\frac{\partial v}{\partial \theta}\right)_{\tau}\right] .
\end{gathered}
$$

Finally, substituting Eq. (A4) into Eq. (A6) gives

$$
\left(\frac{\partial \widehat{\rho}_{f}}{\partial \theta}\right)_{z}=\frac{u^{2}+v^{2}}{u p_{u}-v p_{v}}\left[p_{u}\left(\frac{\partial v}{\partial \theta}\right)_{\tau}-p_{v}\left(\frac{\partial u}{\partial \theta}\right)_{\tau}\right],
$$

where $u, v, p_{u}, p_{v}, \frac{\partial u}{\partial \theta}$, and $\frac{\partial v}{\partial \theta}$ can be obtained by numerically solving the equations of motions and four additional equations, Eqs. (32)-(35). 
The expression in the square brackets on the right side of Eq. (A7) is just the two-dimensional Jacobian $J_{2}(\tau, \theta)$. It is readily seen from Fig. 3 that $\left(\frac{\partial \widehat{\rho}_{f}}{\partial \theta}\right)_{z}=0$ at the extrema of $\rho_{f}$, and, therefore, $J_{2}(\tau, \theta)$ goes through zero (i.e., the classical amplitude goes to infinity therein). In other words, caustics are singularities.
[1] Yu. N. Demkov, V. D. Kondratovich, and V. N. Ostrovsky, JETP Lett. 34, 403 (1981).

[2] V. D. Kondratovich and V. N. Ostrovsky, J. Phys. B 17, 1981 (1984); 17, 2011 (1984).

[3] C. Blondel, C. Delsart, and F. Dulieu, Phys. Rev. Lett. 77, 3755 (1996).

[4] C. Blondel, C. Delsart, F. Dulieu, and C. Valli, Eur. Phys. J. D 5, 207 (1997).

[5] T. Kramer, C. Bracher, and M. Kleber, J. Phys. A 35, 8361 (2002).

[6] C. Valli, C. Blondel, and C. Delsart, Phys. Rev. A 59, 3809 (1999).

[7] C. Blondel, C. Delsart, and F. Goldfarb, J. Phys. B 34, L281 (2001).

[8] C. Blondel, W. Chaibi, C. Delsart, C. Drag, F. Goldfarb, and S. Kröger, Eur. Phys. J. D 33, 335 (2005).

[9] T. Anderson, Phys. Rep. 394, 157 (2004).

[10] M. L. Du, Phys. Rev. A 40, 4983 (1989).

[11] C. Bracher and J. B. Delos, Phys. Rev. Lett. 96, 100404 (2006); C. Bracher, T. Kramer, and J. B. Delos, Phys. Rev. A 73, 062114 (2006).
[12] S. Gao, G. C. Yang, S. L. Lin, and M. L. Du, Eur. Phys. J. D 42, 189 (2007).

[13] C. Nicole, H. L. Offerhaus, M. J. J. Vrakking, F. Lépine, and C. Bordas, Phys. Rev. Lett. 88, 133001 (2002).

[14] Ch. Bordas, F. Lépine, C. Nicole, and M. J. J. Vrakking, Phys. Rev. A 68, 012709 (2003).

[15] M. L. Du and J. B. Delos, Phys. Rev. Lett. 58, 1731 (1987); M. L. Du and J. B. Delos, Phys. Rev. A 38, 1896 (1988); 38, 1913 (1988).

[16] J. Gao and J. B. Delos, Phys. Rev. A 46, 1455 (1992).

[17] A. Alijah and A. von Hopffgarten, J. Phys. B 27, 843 (1994).

[18] G. Alvarez, R. J. Damburg, and H. J. Silverstone, Phys. Rev. A 44, 3060 (1991).

[19] M. S. Child, Semiclassical Mechanics with Molecular Application (Clarendon, Oxford, UK, 1991).

[20] M. Abramowitz and I. E. Stegun, Handbook of Mathematical Functions (Department of Commerce, Washington, DC, 1972).

[21] L. B. Zhao and J. B. Delos, Phys. Rev. A 81, 053418 (2010). 